CUBO A Mathematical Journal Vol.13, $N^{\underline{O}} 03$, (1-15). October 2011

\title{
On the semilocal convergence of Newton-type methods, when the derivative is not continuously invertible
}

\author{
IOANNIS K. ARgYros \\ Department of Mathematics Sciences, \\ Cameron University, \\ Lawton, OK 73505, USA. \\ email: iargyros@cameron.edu \\ and \\ SAÏD HILOUT \\ Laboratoire de Mathématiques et Applications, \\ Poitiers University, \\ Bd. Pierre et Marie Curie, Téléport 2, B.P. 30179 \\ 86962 Futuroscope Chasseneuil Cedex, France \\ email: said.hilout@math. univ--poitiers.fr
}

\begin{abstract}
We provide a semilocal convergence analysis for Newton-type methods to approximate a locally unique solution of a nonlinear equation in a Banach space setting. The Fréchetderivative of the operator involved is not necessarily continuous invertible. This way we extend the applicability of Newton-type methods [1]-[12].

We also provide weaker sufficient convergence conditions, and finer error bound on the distances involved (under the same computational cost) than [1]-[12], in some intersting cases. Numerical examples are also provided in this study.
\end{abstract}




\section{RESUMEN}

Ofrecemos un análisis de convergencia semilocal de los métodos de Newton type para aproximar una solución local única de una ecuación no lineal en un entorno de un espacio de Banach. L derivada de Fréchet del operador en cuestión no es necesariamente invertible continua. De esta manera ampliamos la aplicabilidad de los métodos del tipo Newton [1]- 12].

También proporcionamos condiciones suficientes mas dbiles de convergencia, y una cota de error más fina de las distancias involucradas que [1]-12] (en el mismo coste computacional), en algunos casos interesantes. también presentamos ejemplos numéricos.

Keywords: Newton-type methods, Banach space, small divisors, non-invertible operators, semilocal convergence, Newton-Kantorovich-type hypothesis.

Mathematics Subject Classification: 65H10, 65G99, 65J15, 47H17, 49M15.

\section{Introduction}

In this study we are concerned with the problem of approximating a locally unique solution $x^{\star}$ of equation

$$
F(x)=0 \text {, }
$$

where, $\mathrm{F}$ is a Fréchet-differentiable operator defined on a convex subset $\mathbb{D}$ of a Banach space $\mathcal{X}$ with values in a Banach space $\mathcal{Y}$.

A large number of problems in applied mathematics and also in engineering are solved by finding the solutions of certain equations. For example, dynamic systems are mathematically modeled by difference or differential equations, and their solutions usually represent the states of the systems. For the sake of simplicity, assume that a time-invariant system is driven by the equation $\dot{x}=\mathrm{T}(\mathrm{x})$, for some suitable operator $\mathrm{T}$, where $x$ is the state. Then the equilibrium states are determined by solving equation (1.1). Similar equations are used in the case of discrete systems. The unknowns of engineering equations can be functions (difference, differential, and integral equations), vectors (systems of linear or nonlinear algebraic equations), or real or complex numbers (single algebraic equations with single unknowns). Except in special cases, the most commonly used solution methods are iterative--when starting from one or several initial approximations a sequence is constructed that converges to a solution of the equation. Iteration methods are also applied for solving optimization problems. In such cases, the iteration sequences converge to an optimal solution of the problem at hand. Since all of these methods have the same recursive structure, they can be introduced and discussed in a general framework. 
The most popular method for generating a sequence $\left\{x_{n}\right\}(n \geq 0)$, approximating $x^{\star}$ is undoubtedly Newton's method:

$$
x_{n+1}=x_{n}-F^{\prime}\left(x_{n}\right)^{-1} F\left(x_{n}\right) \quad(n \geq 0), \quad\left(x_{0} \in \mathbb{D}\right) .
$$

There is an extensive literature on local as well as semilocal convergence results for Newton's method [1]-12].

However, there many problems for which Newton's method is not applicable in its original form. A case of interest occurs when the derivative is not continuously invertible, as for instance, dealing with problems involving small divisors, or other important examples [4], 6]-[10]. Several Newton-type methods have addressed this problem [1]-12. Moret in [10] unified a large class of such Newton-type methods, where, at each step, the inverse of the derivative, is replaced by a linear operator, which is obtained recursively from the previous one.

Two iterative schemes were provided in [10]:

Scheme 1. Let the following be given: a Banach space $\mathbb{Z}$, an operator valued mapping $\mathcal{B}: \mathbb{D} \longrightarrow$ $\mathcal{L}(\mathcal{X}, \mathcal{Y}), x_{0} \in \mathbb{D}, \mathcal{S}_{-1} \in \mathcal{L}(\mathbb{Z}, \mathcal{Y}), \mathcal{R}_{-1} \in \mathcal{L}(\mathbb{Z}, \mathcal{X})$.

For $n \geq 0$, let $\mathrm{N}_{\mathrm{n}} \in \mathcal{L}(\mathbb{Z}, \mathbb{Z})$, and set:

$$
\begin{aligned}
& \mathcal{S}_{\mathrm{n}}=\mathcal{S}_{\mathrm{n}-1} \mathrm{~N}_{\mathrm{n}}+\mathcal{B}\left(\mathrm{x}_{\mathrm{n}}\right) \mathcal{R}_{\mathrm{n}-1}, \\
& \mathcal{R}_{\mathrm{n}}=\mathcal{R}_{\mathrm{n}-1}+\mathcal{R}_{\mathrm{n}-1} \mathrm{~N}_{\mathrm{n}}, \\
& x_{\mathrm{n}+1}=\mathrm{x}_{\mathrm{n}}+\mathcal{R}_{\mathrm{n}} \Delta_{\mathrm{n}},
\end{aligned}
$$

$\Delta_{\mathrm{n}}$ being a possible approximate solution of

$$
\mathcal{S}_{\mathrm{n}} \Delta_{\mathrm{n}}=-\mathrm{F}\left(\mathrm{x}_{\mathrm{n}}\right)
$$

That is $\Delta_{\mathrm{n}}$ satisfies an equation of type

$$
\mathcal{S}_{\mathrm{n}} \Delta_{\mathrm{n}}=-\left(\mathrm{F}\left(\mathrm{x}_{\mathrm{n}}\right)+\mathrm{r}_{\mathrm{n}}\right)
$$

for a suitable null sequence $\left\{r_{n}\right\} \subset \mathcal{Y}$.

Moret [10] provided a semilocal convergence analysis for Newton's method under general conditions on the mapping $\mathcal{B}$, the starting guesses $\chi_{0}, \mathcal{S}_{-1}, \mathcal{R}_{-1}$, the operators $\mathrm{N}_{n}$, and the sequence $\left\{r_{n}\right\}$.

Similar results were given for scheme 2 . 
There are two type of problems common to both schemes: the convergence may be too slow, and the schemes may not even be applicable. For example in both schemes a condition of the form:

$$
\left\|\mathcal{I}_{\mathcal{Y}}-\mathcal{T} \mathcal{R}_{-1}\right\|<1
$$

is required, where,

$$
\mathcal{T}=\mathrm{F}^{\prime}\left(\mathrm{x}_{0}\right) \quad(\text { Scheme } 1)
$$

or

$$
\mathcal{T}=\mathcal{B}\left(x_{0}\right) \quad(\text { Scheme } 2)
$$

There are simple numerical examples to show that condition (1.3) is violated.

Let scalar function $F$ given by:

$$
F(x)=\frac{1}{128} x^{2}-\frac{1}{64} \cos x
$$

and choose $x_{0}=0$.

Then $F^{\prime}\left(x_{0}\right)=0$, which shows that (1.3) is violated for $\mathcal{T}=\mathcal{B}\left(x_{0}\right)=F^{\prime}\left(x_{0}\right)$. Hence, Schemes 1 and 2 cannot be used to approximate $x^{\star}$. In particular, the classical Newton-Moser method [4], [6]-[10], obtained from Scheme 1 , by setting $\mathbb{Z}=\mathcal{Y}, \mathcal{B}=\mathrm{F}^{\prime}, \mathcal{S}_{-1}=\mathcal{I}_{\mathcal{Y}}, \mathrm{N}_{\mathrm{n}}=\mathcal{I}_{\mathcal{Y}}-\mathrm{F}^{\prime}\left(\mathrm{x}_{\mathrm{n}}\right) \mathcal{R}_{\mathrm{n}-1}$, and $r_{n}=0(n \geq 0)$ cannot be used.

To address these problems, we consider the Newton-type methods of the form (NTM):

$$
x_{n+1}=x_{n}-\left(F^{\prime}\left(x_{n}\right)-\mathcal{A}\right)^{-1} F\left(x_{n}\right)
$$

or more generally (NLM):

$$
x_{n+1}=x_{n}-\left(\mathcal{C}\left(x_{n}\right)-\mathcal{A}\right)^{-1} \mathrm{~F}\left(x_{n}\right),
$$

where, $\mathcal{C}(x) \in \mathcal{L}(\mathcal{X}, \mathcal{Y})$ is an approximation of $\mathrm{F}^{\prime}(x),(x \in \mathbb{D})$, and $\mathcal{A}$ a given linear operator.

In the case of function $\mathrm{F}$ given in (1.6) (NTM) can be used to approximate $x^{\star}$, if $\mathcal{A}$ is an invertible operator.

Methods (1.7), and (1.8) can be combined into one, even more general (GNTM):

$$
x_{n+1}=x_{n}-\mathcal{A}\left(x_{n}\right)^{-1} F\left(x_{n}\right)
$$

where, $\mathcal{A}(x) \in \mathcal{L}(\mathcal{X}, \mathcal{Y}),(x \in \mathbb{D})$. 
Note that if:

$$
\mathcal{A}(\mathrm{x})=\mathrm{F}^{\prime}(\mathrm{x})-\mathcal{A}
$$

Or

$$
\mathcal{A}(x)=\mathcal{C}(x)-\mathcal{A}
$$

we obtain (1.7), and (1.8), respectively.

Sufficient conditions for semilocal convergence of (GNTM), and estimates on the distances $\left\|x_{n+1}-x_{n}\right\|,\left\|x_{n}-x^{\star}\right\|$ have been given by several authors [1] $-[6]$, 11], [12].

However, in the special case of (1.10) (or (1.11)), we can do better by a direct approach. That is, we can provide (under the same computational cost) weaker sufficient convergence conditions, and finer error estimates on $\left\|x_{n+1}-x_{n}\right\|,\left\|x_{n}-x^{\star}\right\|(n \geq 0)$.

\section{Semilocal convergence analysis of (GNTM)}

Let $\mathrm{L}_{0}>0, \mathrm{~L}>0, \eta>0, \mathrm{a}>0, \mathrm{c}>1$, be given constants.

Set:

$$
\mathrm{b}=\frac{\mathrm{c}}{2}(\mathrm{~L} \eta+2 \mathrm{a}), \quad \mathrm{r}=\frac{\mathrm{c}-1}{\mathrm{c} \mathrm{L}_{0}}
$$

We need the following result on majorizing sequences for (GNTM):

Lemma 2.1. Assume:

$$
c \in\left(1, \frac{1}{a}\right)
$$

and

$$
\eta \leq \frac{(c-1)(1-c a)}{c\left(L_{0}+\frac{c-1}{2} L\right)}
$$

Then, scalar sequence $\left\{\mathrm{t}_{\mathrm{n}}\right\}(\mathrm{n} \geq 0)$ given by

$$
t_{0}=0, \quad t_{1}=\eta, \quad t_{n+2}=t_{n+1}+\frac{L\left(t_{n+1}-t_{n}\right)+2 a}{2\left(1-L_{0} t_{n+1}\right)}\left(t_{n+1}-t_{n}\right)
$$

is non-decreasing, bounded by

$$
t^{\star \star}=\frac{c-1}{c L_{0}}
$$


and converges to its unique least upper bound $t^{\star}$, satisfying:

$$
0 \leq t^{\star} \leq \frac{\eta}{1-b} \leq t^{\star \star}
$$

Moreover the following estimates hold for all $\mathrm{n} \geq 0$ :

$$
t_{n+2}-t_{n+1} \leq b\left(t_{n+1}-t_{n}\right) \leq b^{n+1} \eta
$$

Proof. We shall show using induction on the integer k:

$$
0 \leq t_{k+2}-t_{k+1}=\frac{L\left(t_{k+1}-t_{k}\right)+2 a}{2\left(1-L_{0} t_{k+1}\right)}\left(t_{k+1}-t_{k}\right) \leq b\left(t_{k+1}-t_{k}\right)
$$

and

$$
t_{k+1}<r_{0}
$$

Estimates (2.7), and (2.8) hold for $k=0$, by the initial conditions. Assume (2.7), and (2.8) hold for all $m \leq k$. Then, we have:

$$
\begin{aligned}
0 \leq t_{k+2}-t_{k+1} & \leq b\left(t_{k+1}-t_{k}\right) \\
& \leq b\left(b\left(t_{k}-t_{k-1}\right)\right)=b^{2}\left(t_{k}-t_{k-1}\right) \leq \cdots \\
& \leq b^{k+1} \eta
\end{aligned}
$$

and

$$
\begin{aligned}
t_{k+1} & \leq t_{k}+b^{k} \eta \\
& \leq t_{k-1}+b^{k-1} \eta+b^{k} \eta \\
& \leq t_{1}+b^{1} \eta+\cdots+b^{k} \eta \\
& =\frac{1-b^{k+1}}{1-b} \eta<\frac{\eta}{1-b} \leq r \quad \text { by (2.1), and (2.2), }
\end{aligned}
$$

which complete the induction for (2.7), and (2.8).

Finally, sequence $\left\{t_{n}\right\}$ is non-decreasing, and bounded above by $t^{\star \star}$, and as such it converges to its unique least upper bound $t^{\star}$.

That completes the proof of Lemma 2.1.

We shall show the following semilocal convergence theorem for (GNTM) in the special case, when $\mathcal{A}$ is given by (1.10).

Theorem 2.1. Let $\mathrm{F}: \mathbb{D} \subseteq \mathcal{X} \longrightarrow \mathcal{Y}$ be a Fréchet-differentiable operator, and let $\mathcal{A}(\mathrm{x}) \in \mathcal{L}(\mathcal{X}, \mathcal{Y})$ be given by (1.10). Assume that there exist an open convex subset $\mathbb{D}$ of $\mathcal{X}, x_{0} \in \mathbb{D}$, a bounded 
inverse $\mathcal{A}\left(\mathrm{x}_{0}\right)^{-1}$ of $\mathcal{A}\left(\mathrm{x}_{0}\right)$, and constants $\mathrm{L}_{0}>0, \mathrm{~L}>0, \mathrm{a} \geq 0$, and $\eta>0$, such that for all $x, y \in \mathbb{D}:$

$$
\begin{aligned}
& \left\|\mathcal{A}\left(\mathrm{x}_{0}\right)^{-1} \mathrm{~F}\left(\mathrm{x}_{0}\right)\right\| \leq \eta, \\
& \left\|\mathcal{A}\left(\mathrm{x}_{0}\right)^{-1}\left[\mathrm{~F}^{\prime}(\mathrm{x})-\mathrm{F}^{\prime}(\mathrm{y})\right]\right\| \leq \mathrm{L}\|\mathrm{x}-\mathrm{y}\|, \\
& \left\|\mathcal{A}\left(\mathrm{x}_{0}\right)^{-1}\left[\mathrm{~F}^{\prime}(\mathrm{x})-\mathcal{A}(\mathrm{x})\right]\right\| \leq \mathrm{a}, \\
& \left\|\mathcal{A}\left(\mathrm{x}_{0}\right)^{-1}\left[\mathcal{A}(\mathrm{x})-\mathcal{A}\left(\mathrm{x}_{0}\right)\right]\right\| \leq \mathrm{L}_{0}\left\|\mathrm{x}-\mathrm{x}_{0}\right\|, \\
& \overline{\mathrm{u}}\left(\mathrm{x}_{0}, \mathrm{t}^{\star}\right)=\left\{\mathrm{x} \in \mathcal{X},\left\|\mathrm{x}-\mathrm{x}_{0}\right\| \leq \mathrm{t}^{\star}\right\} \subseteq \mathbb{D},
\end{aligned}
$$

and

the hypotheses of Lemma 2.1 hold.

Then, sequence $\left\{\mathrm{x}_{\mathrm{n}}\right\}(\mathrm{n} \geq 0)$ generated by $(G N T M)$ is well defined, remains in $\overline{\mathrm{u}}\left(\mathrm{x}_{0}, \mathrm{t}^{\star}\right)$ for all $\mathrm{n} \geq 0$, and converges to a solution $\mathrm{x}^{\star}$ of equation $\mathrm{F}(\mathrm{x})=0$ in $\overline{\mathrm{U}}\left(\mathrm{x}_{0}, \mathrm{t}^{\star}\right)$.

Moreover, the following estimates hold for all $\mathrm{n} \geq 0$ :

$$
\left\|x_{n+1}-x_{n}\right\| \leq t_{n+1}-t_{n},
$$

and

$$
\left\|x_{n}-x^{\star}\right\| \leq t^{\star}-t_{n},
$$

where, sequence $\left\{t_{\mathfrak{n}}\right\}(\mathrm{n} \geq 0)$, and $\boldsymbol{t}^{\star}$ are given in Lemma 2.1.

Furthermore, the solution $x^{\star}$ of equation (1.1) is unique in $\overline{\mathrm{u}}\left(\mathrm{x}_{0}, \mathrm{t}^{\star}\right)$ provided that:

$$
\left(\frac{\mathrm{L}}{2}+\mathrm{a}+\mathrm{L}_{0}\right) \mathrm{t}^{\star}+\mathrm{a}<1 .
$$

Proof. We shall show using induction on $m \geq 0$ :

$$
\left\|x_{m+1}-x_{m}\right\| \leq t_{m+1}-t_{m}
$$

and

$$
\overline{\mathrm{u}}\left(x_{\mathrm{m}+1}, t^{\star}-t_{m+1}\right) \subseteq \overline{\mathrm{u}}\left(x_{m}, t^{\star}-t_{m}\right) .
$$

For every $z \in \overline{\mathrm{u}}\left(\mathrm{x}_{1}, \mathrm{t}^{\star}-\mathrm{t}_{1}\right)$,

$$
\begin{aligned}
\left\|z-x_{0}\right\| & \leq\left\|z-x_{1}\right\|+\left\|x_{1}-x_{0}\right\| \\
& \leq t^{\star}-t_{1}+t_{1}=t^{\star}-t_{0},
\end{aligned}
$$


implies $z \in \overline{\mathrm{U}}\left(\mathrm{x}_{0}, \mathrm{t}^{\star}-\mathrm{t}_{0}\right)$. We also have

$$
\left\|x_{1}-x_{0}\right\|=\left\|\mathcal{A}\left(x_{0}\right)^{-1} F\left(x_{0}\right)\right\| \leq \eta=t_{1}-t_{0} .
$$

That is (2.17), and (2.18) hold for $m=0$.

Given they hold for $n \leq m$, then:

$$
\begin{aligned}
\left\|x_{m+1}-x_{0}\right\| & \leq \sum_{\substack{i=1 \\
m+1}}^{m+1}\left\|x_{i}-x_{i-1}\right\| \\
& \leq \sum_{i=1}^{m+1}\left(t_{i}-t_{i-1}\right)=t_{m+1}-t_{0}=t_{m+1}
\end{aligned}
$$

and

$$
\begin{aligned}
\left\|x_{m}+\theta\left(x_{m+1}-x_{m}\right)-x_{0}\right\| & \leq t_{m}+\theta\left(t_{m+1}-t_{m}\right) \\
& \leq t^{\star}
\end{aligned}
$$

for all $\theta \in(0,1)$.

Using (2.8), (2.12), the induction hypotheses, we get:

$$
\begin{aligned}
\left\|\mathcal{A}\left(\mathrm{x}_{0}\right)^{-1}\left[\mathcal{A}\left(\mathrm{x}_{\mathrm{m}+1}\right)-\mathcal{A}\left(\mathrm{x}_{0}\right)\right]\right\| & \leq \mathrm{L}_{0}\left\|\mathrm{x}_{\mathrm{m}+1}-\mathrm{x}_{0}\right\| \\
& \leq \mathrm{L}_{0}\left(\mathrm{t}_{\mathrm{m}+1}-\mathrm{t}_{0}\right) \\
& \leq \mathrm{L}_{0} \mathrm{t}_{\mathrm{m}+1}<1 .
\end{aligned}
$$

It follows from (2.19), and the Banach lemma on invertible operators [4], that $\mathcal{A}\left(\mathrm{x}_{\mathrm{m}+1}\right)^{-1}$ exists, and

$$
\left\|\mathcal{A}\left(\mathrm{x}_{\mathrm{m}+1}\right)^{-1} \mathcal{A}\left(\mathrm{x}_{0}\right)\right\| \leq\left(1-\mathrm{Lt}_{\mathrm{m}+1}\right)^{-1} .
$$

Using (1.9), we obtain the approximation:

$$
\begin{aligned}
x_{m+2}-x_{m+1}= & -\mathcal{A}\left(x_{m+1}\right)^{-1} F\left(x_{m+1}\right) \\
= & -\mathcal{A}\left(x_{m+1}\right)^{-1} \mathcal{A}\left(x_{0}\right) \mathcal{A}\left(x_{0}\right)^{-1} \\
& \left(\int_{0}^{1}\left[F^{\prime}\left(x_{m+1}+\theta\left(x_{m}-x_{m+1}\right)\right)-F^{\prime}\left(x_{m}\right)\right]\left(x_{m+1}-x_{m}\right) d \theta+\right. \\
& \left.\left(F^{\prime}\left(x_{m}\right)-\mathcal{A}\left(x_{m}\right)\right)\left(x_{m+1}-x_{m}\right)\right)
\end{aligned}
$$

Using (2.10)-(2.12), (2.17) (2.20), and (2.21), and the induction hypotheses, we obtain in turn: 


$$
\begin{aligned}
\left\|x_{m+2}-x_{m+1}\right\| & \leq\left(1-L_{0} t_{m+1}\right)^{-1}\left(\frac{L}{2}\left\|x_{m+1}-x_{m}\right\|^{2}+a\left\|x_{m+1}-x_{m}\right\|\right) \\
& \leq\left(1-L_{0} t_{m+1}\right)^{-1}\left(\frac{L}{2}\left(t_{m+1}-t_{m}\right)+a\right)\left(t_{m+1}-t_{m}\right) \\
& =t_{m+2}-t_{m+1},
\end{aligned}
$$

which shows (2.17) for all $m \geq 0$.

Thus, for every $z \in \overline{\mathrm{u}}\left(\mathrm{x}_{\mathrm{m}+2}, \mathrm{t}^{\star}-\mathrm{t}_{\mathrm{m}+2}\right)$, we have:

$$
\begin{aligned}
\left\|z-x_{m+1}\right\| & \leq\left\|z-x_{m+2}\right\|+\left\|x_{m+2}-x_{m+1}\right\| \\
& \leq t^{\star}-t_{m+2}+t_{m+2}-t_{m+1}=t^{\star}-t_{m+1},
\end{aligned}
$$

which shows (2.18) for all $m \geq 0$.

Lemma 2.1 implies that sequence $\left\{t_{n}\right\}$ is Cauchy. Moreover, it follows from (2.17) and (2.18) that $\left\{x_{n}\right\}(n \geq 0)$ is also a Cauchy sequence in a Banach space $\mathcal{X}$, and as such it converges to some $x^{\star} \in \overline{\mathrm{U}}\left(\mathrm{x}_{0}, \mathrm{t}^{\star}\right)$ (since $\overline{\mathrm{U}}\left(\mathrm{x}_{0}, \mathrm{t}^{\star}\right)$ is a closed set).

By letting $\mathrm{m} \longrightarrow \infty$ in (2.22), we obtain $\mathrm{F}\left(\mathrm{x}^{\star}\right)=0$. Furthermore estimate (2.15) is obtained from (2.14) by using standard majorization techniques [1, 4. Finally to show that $x^{\star}$ is the unique solution of equation (1.1) in $\overline{\mathrm{u}}\left(\mathrm{x}_{0}, \mathrm{t}^{\star}\right)$, as in (2.21) and (2.22), we get in turn for $\mathrm{y}^{\star} \in \overline{\mathrm{u}}\left(\mathrm{x}_{0}, \mathrm{t}^{\star}\right)$, with $\mathrm{F}\left(\mathrm{y}^{\star}\right)=0$, the estimation:

$$
\begin{aligned}
\left\|y^{\star}-x_{m+1}\right\| \leq & \left\|\mathcal{A}\left(x_{m}\right)^{-1} \mathcal{A}\left(x_{0}\right)\right\| \\
& \left(\int_{0}^{1}\left\|\mathcal{A}\left(x_{0}\right)^{-1}\left(F^{\prime}\left(x_{m}+\theta\left(y^{\star}-x_{m}\right)\right)-F^{\prime}\left(x_{m}\right)\right)\right\| d \theta\right. \\
& \left.+\left\|\mathcal{A}\left(x_{0}\right)^{-1}\left[F^{\prime}\left(x_{m}\right)-\mathcal{A}\left(x_{m}\right)\right]\right\|\right)\left\|y^{\star}-x_{m}\right\| \\
\leq & \left(1-L_{0} t_{m+1}\right)^{-1}\left(\frac{L}{2}\left\|y^{\star}-x_{m}\right\|^{2}+a\left\|y^{\star}-x_{m}\right\|\right) \\
\leq & \left(1-L_{0} t_{m+1}\right)^{-1}\left(\frac{L}{2}\left(t^{\star}-t_{m}\right)+a\right)\left\|y^{\star}-x_{m}\right\| \\
\leq & \left(1-L_{0} t^{\star}\right)^{-1}\left(\frac{L}{2}\left(t^{\star}-t_{0}\right)+a\right)\left\|x^{\star}-x_{m}\right\| \\
< & \left\|y^{\star}-x_{m}\right\|
\end{aligned}
$$

by $(2.20)$. $x^{\star}=y^{\star}$.

It follows by (2.23) that $\lim _{\mathrm{m} \longrightarrow \infty} x_{\mathrm{m}}=\mathrm{y}^{\star}$. But we showed $\lim _{\mathrm{m} \longrightarrow \infty} x_{\mathrm{m}}=x^{\star}$. Hence, we deduce 
That completes the proof of Theorem 2.1.

Note that $t^{\star}$ can be replaced by $t^{\star \star}$ given by (2.4) in the uniqueness hypothesis provided that $\overline{\mathrm{U}}\left(x_{0}, t^{\star \star}\right) \subseteq \mathbb{D}$, or in all hypotheses of the theorem.

\section{Applications}

Remark 3.1. According to [4], [6], [12], the sufficient convergence condition becomes

$$
\eta \leq \frac{(1-a)^{2}}{2 L}
$$

and the majorizing iteration $\left\{v_{n}\right\}$ is given by:

$$
v_{0}=0, \quad v_{1}=\eta, \quad v_{n+2}=v_{n+1}+\frac{\frac{L}{2} v_{n+1}^{2}-(1-a) v_{n+1}+\eta}{1-L_{0} v_{n+1}} \quad(n \geq 0) .
$$

In view of (2.2), and (3.1), our condition is weaker, if:

$$
\frac{(1-a)^{2}}{2 L}<\frac{(c-1)(1-c a)}{c\left(L_{0}+\frac{c-1}{2} L\right)}
$$

Let $\mathrm{L}_{0}=\mathrm{p} \mathrm{L}$, for $\mathrm{p} \in[0,1]$.

Then (3.3) holds, if:

$$
\frac{(1-a)^{2}}{2}<\frac{(c-1)(1-c a)}{c\left(p+\frac{c-1}{2}\right)}
$$

If $\mathrm{p}$ is close enough to zero, and e.g. $\mathrm{c}=2$, we have (3.4) holds provided:

$$
(1-a)^{2}<2(1-2 a)
$$

or

$$
a \in(0, \sqrt{2}-1)
$$

As example, set $\mathrm{p}=.1, \mathrm{c}=2, \mathrm{a}=.3$, then condition 3.4) becomes:

$$
.245<.333
$$

Hence, for $\eta \in(.245, .333)$, our results can apply, where as the ones in [4], [6], [12] cannot guarantee convergence, since (3.1) is violated. 
Concerning the error estimates, if:

$$
\frac{\left(L-2 L_{0}\right) \eta+2(a+1)}{1-L_{0} \eta}(1-\eta)+L \eta<1+2 a,
$$

then, we have

$$
v_{0}=t_{0}, \quad v_{1}=t_{1}, \quad v_{2}=t_{2}, \quad t_{3}-t_{2}<v_{3}-v_{2} \text {. }
$$

An inductive argument shows:

$$
\begin{gathered}
t_{n}<v_{n} \quad(n \geq 3), \\
t_{n+1}-t_{n}<v_{n+1}-v_{n} \quad(n \geq 2), \\
t^{\star}-t_{n}<v^{\star}-v_{n} \quad(n \geq 2),
\end{gathered}
$$

and

$$
t^{\star}<v^{\star}
$$

Estimates (3.6) holds, for example, let

$$
\mathrm{L}_{0}=.00005, \quad \mathrm{~L}=.0001, \quad \eta=.8, \quad \mathrm{a}=.0001
$$

to obtain

$$
.400496017<1.0002 \text {. }
$$

Note also that (2.2), and (3.1) also hold, since:

$$
\eta \leq 4999
$$

and

$$
\eta \leq 4999.00005
$$

respectively.

Hence, the claims made in the introduction of this study are now justified.

In practice, on will test (2.2), (3.1), (3.6), and then use the combination of the best results.

Let return back to the numerical examples in the introduction of this study.

Example 3.1. Let $\mathbb{D}=[-4,4]$, and choose $\mathrm{c}=2$, and $\mathrm{a}=.2$. Then, we have:

$$
\mathrm{L}_{\mathrm{o}}=\mathrm{L}=\frac{5}{32}, \quad \eta=\frac{5}{64}
$$

and (2.2) becomes:

$$
.15625<1.28 \text {. }
$$

Hence, the conclusions of Theorem 2.1 apply to solve equation $\mathrm{F}(\mathrm{x})=0$. 
Example 3.2. Define the scalar function $\mathrm{F}$ by $\mathrm{F}(\mathrm{x})=\mathrm{c}_{0} \mathrm{x}+\mathrm{c}_{1}+\mathrm{c}_{2} \sin \mathrm{e}^{\mathrm{c}_{3} \mathrm{x}}$, $\mathrm{x}_{0}=0$, where $\mathrm{c}_{\mathrm{i}}$, $i=1,2,3$ are given parameters. Then it can easily be seen that for $\mathrm{c}_{3}$ large and $\mathrm{c}_{2}$ sufficiently small, $\frac{\mathrm{L}}{\mathrm{L}_{0}}$ can be arbitrarily large. That is (2.2) may be satisfied but not (3.1).

We provide two examples, where $\mathrm{L}_{0}<\mathrm{L}$.

Example 3.3. Let $\mathcal{X}=\mathcal{Y}=\mathcal{C}[0,1]$ be the space of real-valued continuous functions defined on the interval $[0,1]$ with norm

$$
\|x\|=\max _{0 \leq s \leq 1}|x(s)| .
$$

Let $\theta \in[0,1]$ be a given parameter. Consider the "Cubic" integral equation:

$$
u^{3}(s)+\lambda u(s) \int_{0}^{1} q(s, t) u(t) d t+y(s)-\theta=0
$$

Here the kernel $\mathrm{q}(\mathrm{s}, \mathrm{t})$ is a continuous function of two variables defined on $[0,1] \times[0,1]$; the parameter $\lambda$ is a real number called the "albedo" for scattering; $\mathrm{y}(\mathrm{s})$ is a given continuous function defined on $[0,1]$ and $x(\mathrm{~s})$ is the unknown function sought in $\mathcal{C}[0,1]$. Equations of the form (3.8) arise in the kinetic theory of gasses [4], [5]. For simplicity, we choose $\mathrm{u}_{0}(\mathrm{~s})=0, \mathrm{y}(\mathrm{s})=1$, and $\mathrm{q}(\mathrm{s}, \mathrm{t})=\frac{\mathrm{s}}{\mathrm{s}+\mathrm{t}}$, for all $\mathrm{s} \in[0,1]$, and $\mathrm{t} \in[0,1]$, with $\mathrm{s}+\mathrm{t} \neq 0$. If we let $\mathbb{D}=\mathrm{u}\left(\mathrm{u}_{0}, 1-\theta\right)$, and define the operator $\mathrm{F}$ on $\mathbb{D}$ by:

$$
F(x(s))=\frac{1}{3000} x^{3}(s)+\lambda x(s) \int_{0}^{1} q(s, t) x(t) d t+y(s)-\theta,
$$

for all $\mathrm{s} \in[0,1]$, then every zero of $\mathrm{F}$ satisfies equation (3.8).

We have the estimates:

$$
\max _{0 \leq s \leq 1}\left|\int \frac{s}{s+t} d t\right|=\ln 2
$$

Therefore, if we set $\mathrm{a}^{-1}=\left\|\mathcal{A}\left(\mathrm{u}_{0}\right)^{-1}\right\|$, then it follows from hypotheses of Theorem 2.1:

$$
\begin{gathered}
F^{\prime}\left(u_{0}(s)\right)=0 \\
\eta=a^{-1}(|\lambda| \ln 2+1-\theta), \\
L=2 a^{-1}\left(|\lambda| \ln 2+\frac{2-\theta}{1000}\right) \quad \text { and } L_{0}=a^{-1}\left(2|\lambda| \ln 2+\frac{3-\theta}{1000}\right) .
\end{gathered}
$$

It follows from Theorem [2.1 that if condition (2.2) holds, then problem (3.8) has a unique solution near $\mathrm{u}_{0}$. This assumption can be weaker than the one given before The Newton-Kantorovich hypothesis (3.1), since $\mathrm{L}_{0}<\mathrm{L}$ for all $\theta \in[0,1]$.

For

$$
\lambda=.001, \quad a=.9, \quad \theta=.9, \quad c=1.1
$$


condition (2.2) becomes:

$$
.111188124<.223204896 \text {. }
$$

Hence, the conclusions of Theorem 2.1 apply to solve equation (3.9).

Example 3.4. Consider the following nonlinear boundary value problem [4]

$$
\left\{\begin{aligned}
u^{\prime \prime} & =-u^{3}-\gamma u^{2} \\
u(0) & =0, \quad u(1)=1
\end{aligned}\right.
$$

It is well known that this problem can be formulated as the integral equation

$$
u(s)=s+\int_{0}^{1} Q(s, t)\left(u^{3}(t)+\gamma u^{2}(t)\right) d t
$$

where, Q is the Green function:

$$
Q(s, t)=\left\{\begin{array}{rr}
t(1-s), & t \leq s \\
s(1-t), & s<t
\end{array}\right.
$$

We observe that

$$
\max _{0 \leq s \leq 1} \int_{0}^{1}|Q(s, t)|=\frac{1}{8}
$$

Let $\mathcal{X}=\mathcal{Y}=\mathcal{C}[0,1]$, with norm

$$
\|x\|=\max _{0 \leq s \leq 1}|x(s)| .
$$

Then problem (3.10) is in the form (1.1), where, $\mathrm{F}: \mathbb{D} \longrightarrow \mathcal{Y}$ is defined as

$$
[\mathrm{F}(\mathrm{x})](\mathrm{s})=x(\mathrm{~s})-\mathrm{s}-\int_{0}^{1} \mathrm{Q}(\mathrm{s}, \mathrm{t})\left(\mathrm{x}^{3}(\mathrm{t})+\gamma \mathrm{x}^{2}(\mathrm{t})\right) \mathrm{dt} .
$$

It is easy to verify that the Fréchet derivative of $\mathrm{F}$ is defined in the form

$$
\left[F^{\prime}(x) v\right](s)=v(s)-\int_{0}^{1} Q(s, t)\left(3 x^{2}(t)+2 \gamma x(t)\right) v(t) d t
$$

If we set $\mathrm{u}_{0}(\mathrm{~s})=\mathrm{s}$, and $\mathbb{D}=\mathrm{U}\left(\mathrm{u}_{0}, \mathrm{R}\right)$, then since $\left\|\mathrm{u}_{0}\right\|=1$, it is easy to verify that $\mathrm{U}\left(\mathrm{u}_{0}, \mathrm{R}\right) \subset \mathrm{U}(0, \mathrm{R}+1)$. It follows that $2 \gamma<5$, then

$$
\begin{gathered}
\left\|I-F^{\prime}\left(u_{0}\right)\right\| \leq \frac{3\left\|u_{0}\right\|^{2}+2 \gamma\left\|u_{0}\right\|}{8}=\frac{3+2 \gamma}{8}, \\
\left\|F^{\prime}\left(u_{0}\right)^{-1}\right\| \leq \frac{1}{1-\frac{3+2 \gamma}{8}}=\frac{8}{5-2 \gamma} \\
\left\|F\left(u_{0}\right)\right\| \leq \frac{\left\|u_{0}\right\|^{3}+\gamma\left\|u_{0}\right\|^{2}}{8}=\frac{1+\gamma}{8}
\end{gathered}
$$




$$
\left\|F\left(u_{0}\right)^{-1} F\left(u_{0}\right)\right\| \leq \frac{1+\gamma}{5-2 \gamma} .
$$

On the other hand, for $x, y \in \mathbb{D}$, we have

$$
\left[\left(F^{\prime}(x)-F^{\prime}(y)\right) v\right](s)=-\int_{0}^{1} Q(s, t)\left(3 x^{2}(t)-3 y^{2}(t)+2 \gamma(x(t)-y(t))\right) v(t) d t .
$$

Consequently,

$$
\begin{aligned}
\left\|F^{\prime}(x)-F^{\prime}(y)\right\| & \leq \frac{\|x-y\|(2 \gamma+3(\|x\|+\|y\|))}{8} \\
& \leq \frac{\|x-y\|\left(2 \gamma+6 R+6\left\|u_{0}\right\|\right)}{8} \\
& =\frac{\gamma+6 R+3}{4}\|x-y\|, \\
\left\|F^{\prime}(x)-F^{\prime}\left(u_{0}\right)\right\| & \leq \frac{\left\|x-u_{0}\right\|\left(2 \gamma+3\left(\|x\|+\left\|u_{0}\right\|\right)\right)}{8} \\
& \leq \frac{\left\|x-u_{0}\right\|\left(2 \gamma+3 R+6\left\|u_{0}\right\|\right)}{8} \\
& =\frac{2 \gamma+3 R+6}{8}\left\|x-u_{0}\right\| .
\end{aligned}
$$

Therefore, conditions of Theorem 2.1 hold with

$$
\eta=\frac{1+\gamma}{5-2 \gamma}, \quad L=\frac{\gamma+6 R+3}{4}, \quad L_{0}=\frac{2 \gamma+3 R+6}{8} .
$$

Note also that $\mathrm{L}_{\mathrm{O}}<\mathrm{L}$.

\section{Conclusion}

We provided a semilocal convergence analysis for (GNTM) method in order to approximate a locally unique solution of an equation in a Banach space, when the derivative of the operator involved is not continuously invertible.

We provided an analysis with the following advantages over the work in [1]-12] weaker sufficient convergence conditions, and larger convergence domain. Note that these advantages are obtained under the same computational cost as in [1]-12]. Numerical examples further validating our results are also provided in this study.

Received: September 2009. Revised: October 2009. 


\section{References}

[1] Argyros, I.K., The theory and application of abstract polynomial equations, St.Lucie/CRC/Lewis Publ. Mathematics series, 1998, Boca Raton, Florida, U.S.A.

[2] Argyros, I.K., On the Newton-Kantorovich hypothesis for solving equations, J. Comput. Appl. Math., 169 (2004), 315-332.

[3] Argyros, I.K., A unifying local-semilocal convergence analysis and applications for two-point Newton-like methods in Banach space, J. Math. Anal. Appl., 298 (2004), 374-397.

[4] Argyros, I.K., Computational theory of iterative methods, Series: Studies in Computational Mathematics, 15, Editors: C.K. Chui and L. Wuytack, Elsevier Publ. Co., New York, USA, 2007.

[5] Chandrasekhar, S., Radiative transfer, Dover Publ., New York, 1960.

[6] Nashed, M.Z., Chen, X., Convergence of Newton-like methods for singular operator equations using outer inverses, Numer. Math., 66 (1993), 235-257.

[7] Craven, B.D., Nashed, M Z., Generalized implicit function theorems when the derivative has no bounded inverse, Nonlinear Anal., 6 (1982), 375-387.

[8] Hald, O.H., On a Newton-Moser type method, Numer. Math., 23 (1975), 411-426.

[9] Jerome, J.W., An adaptive Newton algorithm based on numerical inversion: regularization as postconditioner., Numer. Math., 47 (1985), 123-138.

[10] Moret, I., On a general iterative scheme for Newton-type methods, Numer. Funct. Anal. Optim., 9 (1987-1988), 1115-1137.

[11] Potra, F.A., Sharp error bounds for a class of Newton-like methods, Libertas Mathematica, 5 (1985), 71-84.

[12] Yamamoto, T., A convergence theorem for Newton-like methods in Banach spaces, Numer. Math., 51 (1987), 545-557. 